\title{
On the Hamiltonian Structure and Three-dimensional Instabilities of Rotating Liquid Bridges
}

\author{
Hans Peter Kruse \\ Mathematisches Institut \\ Technische Universität München, Arcisstrasse 21 \\ D-80290 München, GERMANY \\ kruse@mathematik.tu-muenchen.de
}

\author{
Alex Mahalov \\ Department of Mathematics \\ Arizona State University \\ Tempe, AZ 85287, USA \\ alex@taylor.la.asu.edu
}

\author{
Jerrold E. Marsden \\ Control and Dynamical Systems 107-81 \\ California Institute of Technology \\ Pasadena, CA 91125, USA \\ marsden@cds.caltech.edu
}

This version: January 27, 1998

\begin{abstract}
We consider a rotating inviscid liquid drop trapped between two parallel plates. The liquid-air interface is a free surface and the boundaries of the wetted regions in the plates are also free. We assume that the two contact angles at the plates are equal. We present drop shapes that generalize the catenoids, nodoids and unduloids in the presence of rotation. We describe profile curves of these drops and investigate their stability to three-dimensional perturbations. The instabilities are associated with degeneracies of eigenvalues of the corresponding Hamiltonian linear stability problem. We observe that these instabilities are present even in the case when the analogue of the Rayleigh criterion for twodimensional stability is satisfied.
\end{abstract}

\section{Introduction}

The stability and instability of liquid drops between plates, also known as liquid bridges, has received much attention in recent years from both a mathematical and an engineering point of view. Vogel [1987] derived a lower bound for the volume of a stable liquid drop of cylindrical shape at rest. Carter [1988] conjectured that this volume bound for stability of cylindrical drops is actually a lower bound for the volume of an arbitrary, not necessarily cylindrical, stable liquid drop between plates. This conjecture was proved by Finn and Vogel [1992] in the case that the drop meets the upper and the lower plate in equal contact angles. Zhou [1993] generalized the result to drops which meet the plates in arbitrary angles. The proof of Carter's conjecture relies on the fact that the boundary of a drop at rest is a constant mean 
curvature surface, which meets each plate in prescribed angles. These angles are determined by the material properties of the plates and the liquid.

Vogel [1987] showed that a stable static drop is rotationally symmetric. Finn and Vogel [1992] then use the classification of rotationally symmetric constant mean curvature surfaces given by Delaunay [1841]. Such a surface is called an unduloid, catenoid or nodoid if the mean curvature $\kappa$ of the surface satisfies $\kappa>0, \kappa=0$ or $\kappa<0$, respectively. Note that the mean curvature of a surface (as opposed to the Gaussian curvature) does depend on a choice of the normal vector field (compare do Carmo [1976]). In this paper we choose normal vector fields to be directed outward and define the mean curvature in such a way that a sphere has positive mean curvature. Delaunay [1841] showed that profile curves of rotationally symmetric constant mean curvature surfaces can be generated by rolling a conic section along the symmetry axis and tracing one of its foci. Rolling an ellipse yields an unduloid, rolling a parabola gives a catenoid, and rolling a hyperbola leads to a nodoid. To prove Carter's conjecture, Finn and Vogel [1992] investigate the Hessian of the system potential energy in each of the three cases.

Kruse [1993] took up the study of the dynamics of a drop of an ideal fluid between plates. He showed that the equations of motion have a Hamiltonian structure which generalizes the Hamiltonian structure derived by Lewis, Marsden, Montgomery and Ratiu [1986] for free boundary problems in fluid mechanics without capillarity effects. In particular, it is to be noted that if one wishes to derived the equations from a variational principle or a Poisson bracket structure, then one is forced to make the choice of contact angle conditions that we adopt.

Kruse [1993] derived a stability criterion for rigidly rotating cylindrical drops generalizing Vogel's criterion. In particular, a rigidly rotating cylindrical drop is stable with respect to axisymmetric perturbations if

$$
\frac{h^{2}}{\pi^{2} a^{2}}+\frac{\Omega^{2} h^{2} a}{\pi^{2} \gamma}<1
$$

Here $\gamma$ is a surface tension coefficient, $a$ is the radius of the circular cross section of the drop, $\Omega$ is the rotation rate and $h$ is the distance between the plates. In the case $\Omega=0$ this reduces to the criterion of Vogel [1987]. This result is derived by an energy method which is closely related to the energy-momentum method for Hamiltonian systems with symmetries (see Marsden [1992]).

In applications it is essential to examine the robustness of the dynamical behavior of an idealized and usually highly symmetric model with respect to imperfections. In particular, symmetry breaking imperfections in Hamiltonian systems can have dramatic consequences for the stability of the system. The reduction in system symmetry due to an imperfection may result in splitting 'windows', and hence lead to instabilities (see Guckenheimer and Mahalov [1992], Knobloch, Mahalov and Marsden [1994] for additional information and references).

In this paper the Hamiltonian structure of the equations of motion also plays an important role in our stability investigations. We study the spectrum of the linearization of the equations of motion about a uniformly rotating state and its behavior under system symmetry breaking perturbations. We exhibit a new instability 
mechanism for both cylindrical and non-cylindrical rotating liquid drops trapped between two plates. Our approach uses the Hamiltonian structure and the symmetries of the system in a decisive way. We study rotating drops for different values of the control parameters such as volume, curvature, rotation rate and contact angles (i.e., different materials). To demonstrate our techniques in a simple example, we show instabilities to small system symmetry breaking perturbations of a cylindrical drop forming a contact angle $\pi / 2$ with two parallel vertical plates. We find that for certain values of the parameters $h, a, \Omega$ and $\gamma$ satisfying the condition (1.1) guaranteeing $2 \mathrm{D}$ stability, the linearization of the equations about the relative equilibrium (that is, a uniformly rotating state) has double eigenvalues on the imaginary axis. If the symmetry of the system is broken (so that these two modes can couple), then instability becomes possible. Breaking the rotational symmetry of the liquid bridge will move these eigenvalues off the axis resulting in an instability, as in Knobloch, Mahalov and Marsden [1994].

Because of the Hamiltonian structure of the system, the eigenvalues will move to both sides of the imaginary axis, but still linearly destabilizing the system. This mechanism destabilizes even drops which are stable by the energy criteria mentioned above. This means that a rotating liquid drop between two plates might be energetically stable to two-dimensional perturbations, but nevertheless be 'structurally' unstable. We can associate instabilities with the degeneracies caused by two physically distinguishable eigenmodes of the rotating liquid drop having the same eigenfrequency. The above discussion illustrates well the basic point of this paper: system symmetry breaking perturbations of Hamiltonian systems with symmetry can, under the appropriate circumstances, lead to the loss of linear-and hence nonlinear-stability. These instabilities take place whenever the loss of symmetry results in the splitting of double eigenvalues and are important in applications since they often occur on a dynamical time scale.

The paper is organized as follows. In $\S 2$ we formulate the equations of motion and discuss their Hamiltonian structure which is relevant to the stability analysis. In section $\S 3$ we calculate profile curves of rotationally symmetric relative equilibria. These relative equilibria are analogous to the Delaunay curves in the non-rotating case. In $\S 4$ we take up the stability analysis of rotating liquid drops and columns.

\section{The Equations of Motion and their Hamiltonian Struc- ture}

In this section we formulate the equations of motion of a liquid drop trapped between two parallel flat plates. We assume that surface tension acts on the free boundary $\Sigma$ of the drop and adhesion forces act along the contact surfaces $\Sigma_{i}$ between the drop and each plate $P_{i}, i=0,1$. The flow configuration is shown in Figure 1.

Let $h>0$ denote the distance between the two plates. Choose a cartesian coordinate system $x, y, z$ such that the lower plate $P_{0}$ lies in the $x y$-plane and the $z$-axis points from the lower plate to the upper plate. Let $\mathbf{e}_{z}$ denote the unit vector along the $z$-axis. The equations of motion for an ideal inviscid fluid drop with 


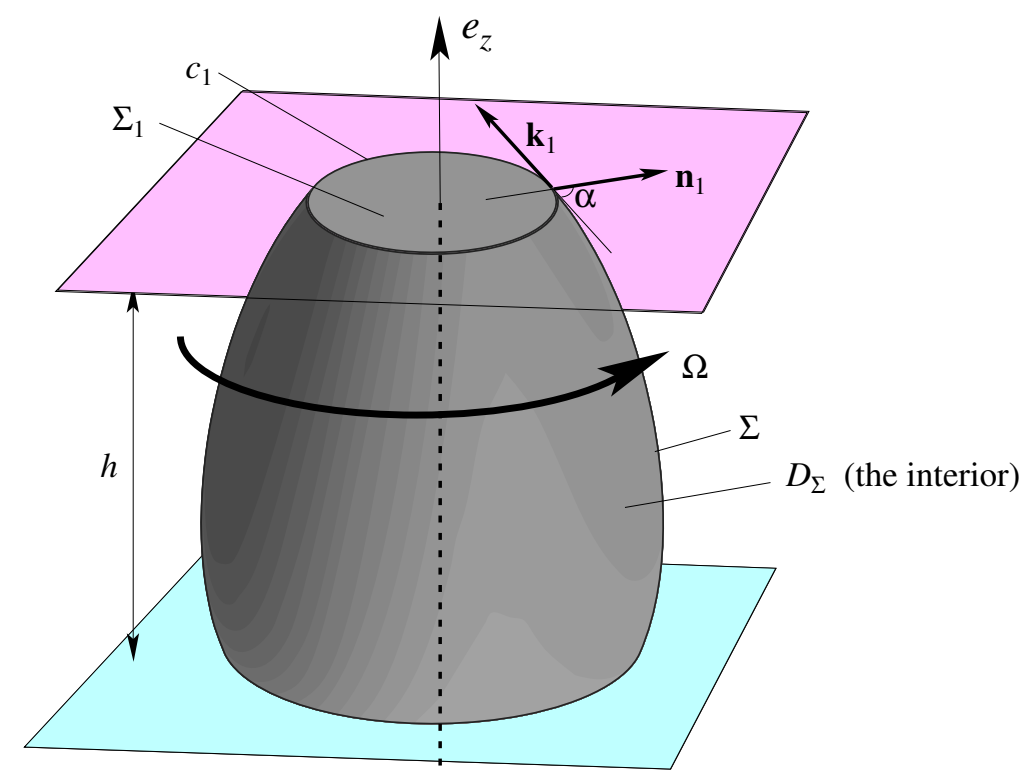

Figure 1: Fluid forming a rotating liquid bridge.

constant density $\rho=1$ trapped between the two plates $P_{0}$ and $P_{1}$ are given by

$$
\begin{aligned}
\frac{\partial \mathbf{v}}{\partial t}+(\mathbf{v} \cdot \nabla) \mathbf{v} & =-\nabla p, & & \\
\nabla \cdot \mathbf{v} & =0, & & \\
\left\langle\mathbf{v}, \mathbf{e}_{z}\right\rangle & =0 & & \text { on } \Sigma_{0} \cup \Sigma_{1}, \\
\frac{\partial \Sigma}{\partial t} & =\langle\mathbf{v}, \mathbf{n}\rangle & & \text { on } \Sigma, \\
p & =\gamma \kappa & & \text { on } \Sigma, \\
\cos \alpha_{i} & =\frac{\sigma_{i}}{\gamma} & & \text { on } c_{i}, \quad i=0,1,
\end{aligned}
$$

where $\mathbf{n}: \Sigma \rightarrow \mathbb{R}^{3}$ denotes an outer unit normal vector field on $\Sigma$, and $\gamma, \kappa$ are, as before, the constant of surface tension and the mean curvature of the surface $\Sigma$, respectively. Physical reasoning shows that $\gamma$ has to be positive (compare Landau and Lifshitz, [1958], §139). The region $\Sigma_{i}$ is the contact surface between the drop and the plate $P_{i}$ and $c_{i}$ is the curve in which the free surface $\Sigma$ meets $P_{i}$. The constant $\sigma_{i}$ depends on the material of the plate $P_{i}$. The contact angle $\alpha_{i}$ between $P_{i}$ and the drop at a point $x \in c_{i}$ is measured between an outer normal vector field $\mathbf{n}_{i}: c_{i} \rightarrow \mathbb{R}^{3}$ to $c_{i}$ in $P_{i}$ and an outer conormal vector field $\mathbf{k}_{i}: c_{i} \rightarrow \mathbb{R}^{3}$ to the manifold $\Sigma$ at the point $x \in c_{i}$. The vector field $\mathbf{k}_{i}: c_{i} \rightarrow \mathbb{R}^{3}$ satisfies the conditions $\mathbf{k}_{i}(x) \in T_{x} \Sigma$, and $\left\langle\mathbf{k}_{i}(x), \xi\right\rangle=0$ for $\xi \in T_{x} c_{i}$, i.e. $\mathbf{k}_{i}(x)$ is tangential to $\Sigma$ and orthogonal to $c_{i}$. Furthermore, $\mathbf{k}_{i}(x)$ has length 1 and points outward from $\Sigma$.

The boundary condition (2.6) along the contact lines is the same one that is used in the static case. In that case it can be derived from variational principles 
(compare Finn [1986]). In Kruse [1993] it is shown how to derive (2.6) in the dynamic case from a Hamiltonian formulation of the problem. Hocking [1992] gives further arguments in favor of the boundary condition (2.6). There is also some work on the analogous problem for the Navier-Stokes equations in the literature: Puchnachev and Solonnikov [1983] study the plane motion of an viscous incompressible fluid with a free surface between two walls. They use the no-slip boundary condition at the walls, which are modelled as two parallel horizontal lines. The free boundary of the fluid is a curve connecting the two walls and meets each of them in a contact point. The fluid is filling the region between the walls to the left of the free boundary. Pukhnachev and Solonnikov study quasistationary motions of the viscous fluid, i.e. motions which are stationary in a coordinate system moving with constant velocity to the right. They denote by $\omega$ the intersection of a certain small semicircle centered about the lower contact point and the region occupied by the fluid and show that the assumption $\mathbf{v} \in W_{2}^{1}(\omega)$ forces the contact angle to be equal to 0 or to $\pi$. Pukhnachev and Solonnikov point out that the proof of this result only makes use of the no-slip boundary condition at the wall and the kinematic boundary condition at the free surface satisfied by the quasistationary velocity field. The fact that $\mathbf{v}$ is a solution of the Navier-Stokes equations does not enter the proof. In Solonnikov [1995] it is shown, that if one furthermore makes use of the fact that $\mathbf{v}$ ist divergence-free, then the contact angle cannot be equal to 0. (Note that the contact angle in Pukhnachev and Solonnikov [1983] and Solonnikov [1995] is measured between the free surface and the wall in the region occupied by the fluid. Therefore, this angle has the same value as the one measured at the contact point according to our definition of contact angle given above.) One may interpret the results of Pukhnachev and Solonnikov to mean that in general the classical no-slip condition is not the correct boundary condition close to the contact lines. Kröner [1987] replaces the no-slip condition by a certain slip condition and derives existence and regularity result for quasistationary motion of a viscous fluid in the presence of a free boundary and contact lines.

In Kruse [1993] it is shown that the equation of motion can be written in Hamiltonian form

$$
\dot{F}=\{F, H\}
$$

for all $F \in \mathcal{F}$, where $\mathcal{F}$ denotes the class of real valued functions on phase space (i.e. $(\Sigma, v)$-space) possessing functional derivatives with respect to $\Sigma$ and $v$, of the form

$$
\frac{\delta F}{\delta \Sigma}: \Sigma \rightarrow \mathbb{R}, \quad \frac{\delta_{i} F}{\delta \Sigma}: c_{i} \rightarrow \mathbb{R}, \quad i=0,1, \quad \frac{\delta F}{\delta v}: D_{\Sigma} \rightarrow \mathbb{R}^{3}
$$

with $\nabla \cdot \frac{\delta F}{\delta v}=0$, such that

$$
\begin{aligned}
D F \cdot \delta \Sigma & =\int_{\Sigma}\left\langle\frac{\delta F}{\delta \Sigma}, \delta \Sigma\right\rangle d S+\int_{c_{0}}\left\langle\frac{\delta_{0} F}{\delta \Sigma}, \delta \Sigma\right\rangle d s+\int_{c_{1}}\left\langle\frac{\delta_{1} F}{\delta \Sigma}, \delta \Sigma\right\rangle d s \\
D F \cdot \delta v & =\int_{D_{\Sigma}}\left\langle\frac{\delta F}{\delta v}, \delta v\right\rangle d V .
\end{aligned}
$$


Here $c_{0}$ and $c_{1}$ are the curves in which $\Sigma$ meets the upper and the lower plate respectively and $D_{\Sigma}$ is the region occupied by the drop. The Poisson bracket of the Hamiltonian formulation is given by

$$
\begin{aligned}
\left\{F_{1}, F_{2}\right\}= & \int_{D_{\Sigma}}\left\langle\nabla \times v, \frac{\delta F_{1}}{\delta v} \times \frac{\delta F_{2}}{\delta v}\right\rangle d V \\
& +\int_{\Sigma}\left(\left\langle\frac{\delta F_{2}}{\delta v}, n\right\rangle \frac{\delta F_{1}}{\delta \Sigma}-\left\langle\frac{\delta F_{1}}{\delta v}, n\right\rangle \frac{\delta F_{2}}{\delta \Sigma}\right) d S \\
& +\int_{c_{0}}\left(\left\langle\frac{\delta F_{2}}{\delta v}, n\right\rangle \frac{\delta_{0} F_{1}}{\delta \Sigma}-\left\langle\frac{\delta F_{1}}{\delta v}, n\right\rangle \frac{\delta_{0} F_{2}}{\delta \Sigma}\right) d s \\
& +\int_{c_{1}}\left(\left\langle\frac{\delta F_{2}}{\delta v}, n\right\rangle \frac{\delta_{1} F_{1}}{\delta \Sigma}-\left\langle\frac{\delta F_{1}}{\delta v}, n\right\rangle \frac{\delta_{1} F_{2}}{\delta \Sigma}\right) d s .
\end{aligned}
$$

We assume that the drop has a constant density $\rho=1$. The Hamiltonian $H \in \mathcal{F}$ is just the sum of kinetic and potential energy:

$$
H=\frac{1}{2} \int\|v\|^{2} d V+\gamma \int_{\Sigma} d S-\sigma_{0} \int_{\Sigma_{0}} d S-\sigma_{1} \int_{\Sigma_{1}} d S
$$

As explained above, $\Sigma_{j}$ is the area enclosed by the plane curve $c_{j}$ for $j=0,1$.

For a derivation of the equations of motion and the detailed discussion of their Hamiltonian structure compare Kruse [1993]. Note that in the present paper we use the convention of Lewis et al. [1986] to take variations of the free surface $\Sigma$ in the normal direction.

In this paper we are especially interested in drops which are rotationally symmetric with respect to the $z$-axis and which rotate rigidly about this axis with constant angular velocity $\Omega$. For the stability analysis of this type of motion it is convenient to write the equations of motion in a coordinate system that rotates with constant angular velocity $\Omega$ about the $z$-axis. In this case Eq. (2.1) has to be replaced by the equation

$$
\frac{\partial \mathbf{v}}{\partial t}+(\mathbf{v} \cdot \nabla) \mathbf{v}+2 \Omega \mathbf{e}_{z} \times \mathbf{v}=-\nabla p
$$

where $p$ is a modified pressure.

\section{The Shape of Rotating Liquid Drops}

In this section we state some results on the geometry of the free boundary of the drop both in the rotationally symmetric and the general case. Free boundaries of static drops can be characterized as surfaces of constant mean curvature which meet the plates in prescribed angles. We will show how some well known results on the geometry of constant mean curvature surfaces generalize to free boundaries of rigidly rotating drops.

\subsection{Theoretical Considerations}

The profile curves of the free boundary of rotationally symmetric liquid drops can be described as graphs of functions $f:[0, h] \rightarrow \mathbb{R}^{+}$defined on the section of the $z$-axis 
between the plates. If such a drop is rigidly rotating between the plates with angular velocity $\Omega$, the function $f:[0, h] \rightarrow \mathbb{R}^{+}$is a solution of the following boundary value problem

$$
\begin{gathered}
\gamma \kappa-\frac{1}{2} \Omega^{2} f^{2}=c, \\
f^{\prime}(0)=-\frac{\sigma_{0}}{\sqrt{\gamma^{2}-\sigma_{0}^{2}}}, \quad f^{\prime}(h)=\frac{\sigma_{1}}{\sqrt{\gamma^{2}-\sigma_{1}^{2}}},
\end{gathered}
$$

for some real number $c$ (compare Kruse, Marsden, Scheurle [1993]). The prime denotes differentiation with respect to $z$. Note that in the case $\Omega=0$, solutions to these equations represent axially symmetric constant mean curvature surfaces which meet the plates in prescribed angles. We now show how to integrate differential equation (3.1). (For another way to integrate the equations of an axisymmetric rotating drop see Myshkis et al. [1987].)

The rotationally symmetric surface generated by rotating the graph of $f$ about the $z$-axis has mean curvature

$$
\kappa(f)=\frac{1}{f\left(1+f^{\prime 2}\right)^{\frac{1}{2}}}-\frac{f^{\prime \prime}}{\left(1+f^{\prime 2}\right)^{\frac{3}{2}}} .
$$

Substituting (3.3) in (3.1) yields

$$
\gamma \frac{1+f^{\prime 2}-f f^{\prime \prime}}{f\left(1+f^{\prime 2}\right)^{\frac{3}{2}}}-\frac{1}{2} \Omega^{2} f^{2}=c .
$$

Multiply both sides of equation (3.4) by $f f^{\prime}$ to get

$$
\gamma\left(\frac{f}{\left(1+f^{\prime 2}\right)^{\frac{1}{2}}}\right)^{\prime}-\left(\frac{1}{8} \Omega^{2} f^{4}\right)^{\prime}=\left(\frac{c}{2} f^{2}\right)^{\prime} .
$$

Thus, there is $d \in \mathbb{R}$ such that

$$
\gamma \frac{f}{\left(1+f^{\prime 2}\right)^{\frac{1}{2}}}-\frac{1}{8} \Omega^{2} f^{4}-\frac{c}{2} f^{2}=d
$$

Then (up to a factor \pm 1 )

$$
\frac{d f}{d z}=\sqrt{\frac{\gamma^{2} f^{2}}{\left(d+c / 2 f^{2}+\frac{\Omega^{2}}{8} f^{4}\right)^{2}}-1},
$$

so there is $e \in \mathbb{R}$ such that

$$
z(f)=\int_{0}^{f} \frac{d f}{\sqrt{\frac{\gamma^{2} f^{2}}{\left(d+c / 2 f^{2}+\frac{\Omega^{2}}{8} f^{4}\right)^{2}}-1}}+e
$$


for a solution $f=f(z)$ of equation (3.1) (we write $z(f)$ for the inverse of $f(z)$ in the last equation). The constants $c, d, e$ have to be determined such that the boundary conditions (3.2) are satisfied. Obviously, in case $\Omega \neq 0$ and $f$ non-constant, the mean curvature is no longer constant and is given by

$$
\kappa=\frac{c+\frac{1}{2} \Omega^{2} f^{2}}{\gamma}
$$

We now make some remarks on the free boundary of drops which are not necessarily rotationally symmetric. Using ideas of Spruck [1988], we will show how to associate a certain system of partial differential equation to a 'rotating constant mean curvature surface'. In case the rotation rate is zero this is just an ordinary constant mean curvature surface and the system of partial differential equation reduces to the sinh-Gordon equation for a function that determines the metric tensor of the constant mean curvature surface in conformal coordinates. This connection between constant mean curvature surfaces and the sinh-Gordon equation, which is an integrable system, is well known (see Abresch [1987], Bobenko [1991] or Spruck [1988]).

Let $S$ be an arbitrary 2-dimensional surface in $\mathbb{R}^{3}$. If $S$ is at least $C^{2}$, one can locally find conformal coordinates $(X, Y)$ on $S$ (see Spivak [1979]). Let $Z=X+i Y$, then the metric on $S$ induced from the standard metric on $\mathbb{R}^{3}$ is given in these conformal coordinates by

$$
d s^{2}=E|d Z|^{2}=E\left(d X^{2}+d Y^{2}\right)
$$

Let

$$
l d X^{2}+2 m d X d Y+n d Y^{2}
$$

denote the second fundamental form. Let $\kappa$ and $K$ denote mean curvature and Gaussian curvature, respectively. (We use notation of Spruck [1988], with the exception of the mean curvature which is denoted $H$ by Spruck, whereas we use the letter $\kappa$.) Set

$$
\Phi:=l-n-2 i m
$$

Then

$$
K=\kappa^{2}-\frac{|\Phi|^{2}}{4 E^{2}}
$$

One has the Gauss equation (see Spruck [1988])

$$
-K=\frac{\triangle \log E}{2 E}
$$

where $\triangle=\frac{\partial^{2}}{\partial X^{2}}+\frac{\partial^{2}}{\partial Y^{2}}$ denotes the Laplacian. Note, that in conformal coordinates the Laplace-Beltrami operator on $S$ is $\triangle_{L B}=-\frac{1}{E} \triangle$ (compare Jost [1995]). The Codazzi-Mainardi equation is given by

$$
\Phi_{\bar{Z}}=E \kappa_{Z}
$$


Using equations (3.11) and (3.12), one concludes that

$$
\triangle \log E+2 E \kappa^{2}-\frac{|\Phi|^{2}}{2 E}=0 .
$$

Fix a positive constant $g$, the exact value of which will be specified later, and define a function $u$ by

$$
E=g e^{u}
$$

Then equation (3.14) becomes

$$
\triangle u+2 g e^{u} \kappa^{2}-\frac{e^{-u}}{2 g}|\Phi|^{2}=0 .
$$

If $S$ is the free surface of a rigidly rotating liquid drop, one has (assuming, for simplicity, that the surface tension constant $\gamma$ is equal to 1 )

$$
\kappa=c+\frac{1}{2} \Omega^{2} f^{2},
$$

where $c$ is a constant, $\Omega$ denotes the angular velocity and $f$ denotes the distance of the point with coordinates $(X, Y)$ to the rotation axis of the drop. Substituting equation (3.16) into (3.13) and (3.15) leads to

$$
\Phi_{\bar{Z}}=g e^{u} \Omega^{2} f f_{Z}, \quad \triangle u+2 g e^{u}\left(c+\frac{1}{2} \Omega^{2} f^{2}\right)^{2}-\frac{e^{-u}}{2 g}|\Phi|^{2}=0 .
$$

In case $\Omega=0$, it follows from (3.17) that the function $\Phi$ is holomorphic. If, in addition to this, one assumes that $S$ is a torus, then $\Phi$ is forced to be a constant. In this case equations (3.17) reduce to a single differential equation for the variable $u$ alone. If one sets

$$
g:=\frac{|\Phi|}{2 \kappa}
$$

the differential equation for $u$ is given by

$$
\triangle u+\hat{g} \sinh u=0
$$

where

$$
\hat{g}:=2|\Phi| \kappa
$$

Eq. (3.18) is the familiar sinh-Gordon equation for the function which determines the metric tensor of the constant mean curvature surface $S$ in conformal coordinates (see Spruck [1988]). Special families of solutions of these equations corresponding to constant mean curvature tori have been investigated by Abresch [1987] and Bobenko [1991]. Eqs. (3.17) are a generalization of Eq. (3.18) to rotating surfaces. Then solutions of Eq. (3.18) are naturally embedded in a family of solutions 
of Eqs. (3.17) parametrized by $\Omega$. Generalizations of special solutions of Abresch [1987] and Bobenko [1991] to $\Omega \neq 0$ would be of particular interest.

We now show how equation (3.1) can be recovered from equation (3.17) in the case of a rotationally symmetric drop, the boundary $\Sigma$ of which is parametrized by

$$
(\varphi, z) \mapsto(f(z) \cos \varphi, f(z) \sin \varphi, z),
$$

In this case one checks that conformal coordinates on $\Sigma$ are given by

$$
(X, Y)=\left(\varphi, \rho^{-1}(z)\right)
$$

where

$$
\frac{d \rho}{d Y}=\frac{f(\rho(Y))}{\sqrt{1+f^{\prime}(\rho(Y))^{2}}} .
$$

Then, $E=f^{2}$ and putting $g=1$, and defining the function $u$ by $E=e^{u}$, one has

$$
u=2 \ln f .
$$

Thus, equations (3.17) reduce to differential equations in the variables $f$ and $\Phi$. Let $\kappa(f)$ and $K(f)$ be the expressions for the mean curvature and the Gaussian curvature of the surface (3.19), respectively. If we substitute $2 f^{2} \sqrt{\kappa^{2}(f)-K(f)}$ for $|\Phi|$ in the equation $(3.17,2)$, and make use of equation $(3.12)$, equation $(3.17,2)$ reduces to

$$
\kappa^{2}(f)=\left(c+\frac{1}{2} \Omega^{2} f^{2}\right)^{2} .
$$

This equation can be integrated in the way outlined at the beginning of this section.

Thus, equations (3.17) reduce to integrable equations in two different limits: one being the case of a constant mean curvature torus, the other one being the case of a rotating Delaunay surface, which will be studied numerically in the next section. Note that in case of the constant mean curvature torus one has to deal with an integrable partial differential equation whereas a rotating Delaunay surface is described by an ordinary differential equation. It is of great interest to find out if there are other cases, in which our equations (3.17) can be integrated.

\subsection{Profile Curves of Rotating Unduloids, Nodoids and Catenoids}

In this section we construct numerical solutions of Eq. (3.1) considering only the case of equal contact angles $\alpha=\alpha_{1}=\alpha_{2}$. We note that in the case $\Omega=0$ these solutions are the well-known Delaunay curves. Then we study their deformations upon varying the rotation parameter $\Omega$. This leads us to new profile curves which we call rotating Delaunay curves. The surfaces corresponding to these profiles are no longer constant mean curvature surfaces.

For an example of a rotating unduloid, we start with a cylindrical drop with a contact angle $\pi / 2$. Then we continue this solution in two parameters $(\alpha, \Omega)$. We note 


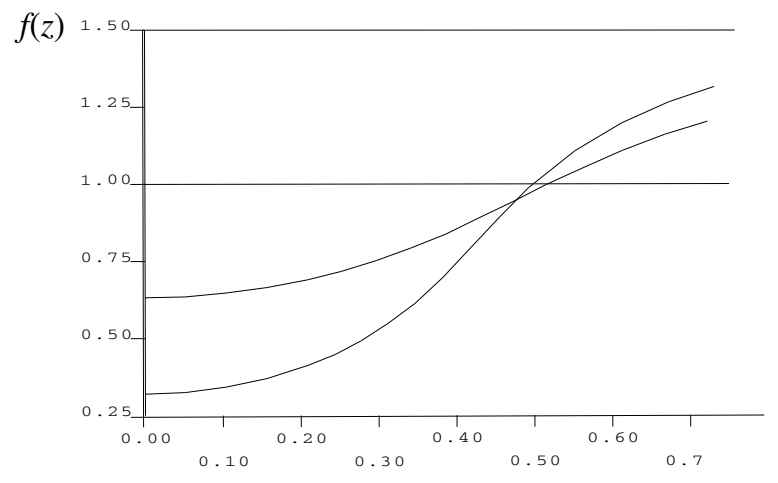

Figure 2: Profile curve of a drop which bifurcates from a cylindrical profile (unduloid) while keeping the contact angle fixed at the value $\pi / 2, \Omega \neq 0$.

that the cylindrical shape is a valid solution for arbitrary rotation rate provided that the contact angle remains $\pi / 2$. However, there are bifurcations from this cylindrical profile for certain values of the rotation rate $\Omega$ and the volume $V$ (see Figure 2).

To study another example of rotating unduloids, we start with a drop at rest represented by a spherical section which belongs to the unduloid family. Again we continue the solution in two parameters $(\alpha, \Omega)$. The corresponding profiles are shown in Figure 3.

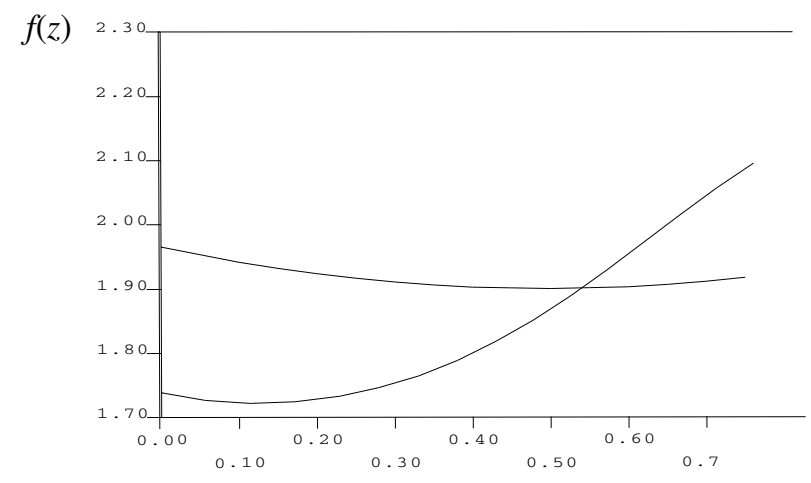

Figure 3: Profile curve of rotating nodoid, $\Omega \neq 0$.

We note that the spherical shape is a valid solution of Eq. (3.1)-(3.2) only for the case $\Omega=0$.

Finally, we start our continuation procedure from a catenoid. We select a representative

$$
z=a \cosh \left(\frac{f}{a}\right)
$$

of the catenoid family. This is an explicit solution for the case $\Omega=0$ (see Finn and 
Vogel [1992]). This solution is continued in two parameters $(\alpha, \Omega)$. The corresponding profiles are shown in Figure 4.

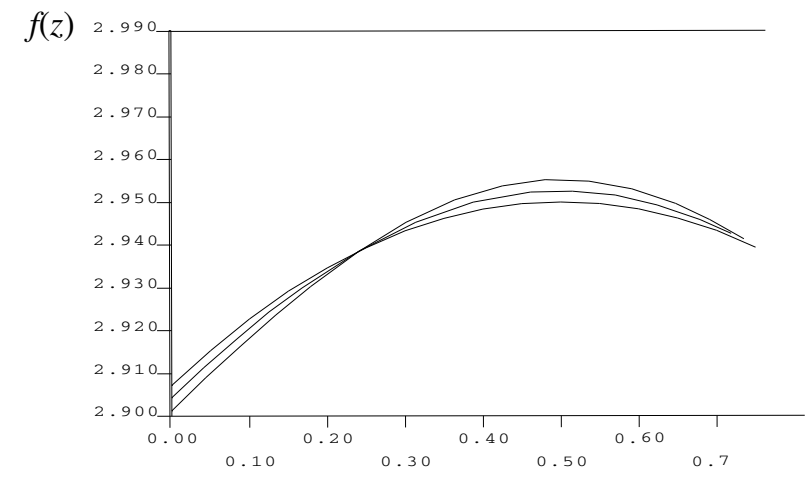

Figure 4: Profile curve of rotating catenoid, $\Omega \neq 0$.

\section{An Example of Three-dimensional Instabilities}

In many problems stability results can be obtained from variational principles using the available conserved quantities (momentum maps or Casimir functions). Such a variational formulation exists for many equations in two-dimensional hydrodynamics, as well as for more general Hamiltonian systems. It allows one to use the conserved quantities to establish the nonlinear stability of equilibria and relative equilibria. The situation is less clear in three-dimensional hydrodynamics. It has been recognized recently that system symmetry breaking perturbations of Hamiltonian systems with symmetry can, under the appropriate circumstances, lead to the loss of stability. These instabilities take place whenever the loss of symmetry results in the splitting of double eigenvalues and are important in applications since they occur on a dynamical time scale. This is, for example, true for the instability of Poiseuille flow in an elliptic pipe which is nearly circular. We refer to Davey and Salwen [1994] and the Appendix to their paper written by P.G. Drazin. This specific problem is generic to a much wider class of fluid dynamical problems which are destabilized when the symmetry group of the dynamical system is reduced. The next step in the analysis is to construct Hamiltonian normal forms near the instability points. We refer to Knobloch, Mahalov and Marsden [1994] where the issue of Hamiltonian normal forms in fluid dynamical problems is addressed.

In this paper we identify a similar instability mechanism for rotating cylindrical liquid bridges. Our analysis predicts 'dangerous' aspect ratios corresponding to geometric shapes more susceptible to three-dimensional instabilities. We extend the analysis of Mahalov [1993] to zero flux boundary conditions in the vertical direction on the vertical boundaries. One only needs to restrict Fourier series to be even in $z$ for horizontal velocities and odd in $z$ for vertical velocity. Such boundary conditions imply zero tangential stress on the vertical boundaries (e.g. Drazin and Reid, 1981). 
There are a number of ways to destabilize the base state (rotationally symmetric rotating liquid bridge) via splitting of the double eigenvalues of the linear stability problem. One possibility is the gyroscopic destabilization by an external Coriolis force (precession). Flow configuration is shown in Figure 5.

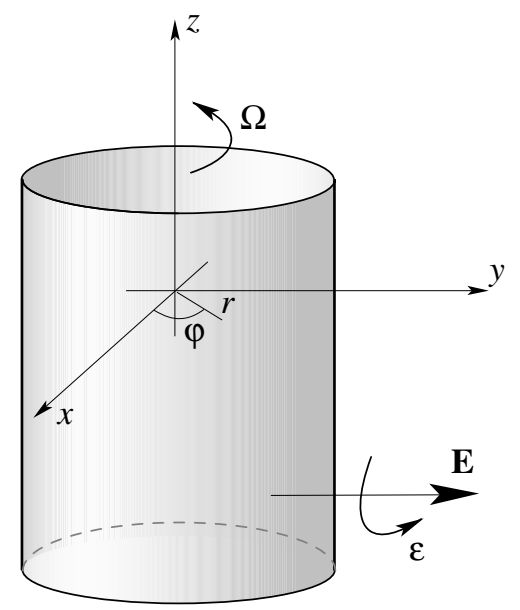

Figure 5: Flow configuration for a rotating liquid bridge subjected to an external Coriolis force.

The coordinate system introduced at the beginning of Section 2 specializes to the cylindrical coordinate system (up to a translation in the $r$ variable by the radius $a$ of the cylinder) $(r, \phi, z)$ where $r$ is the radial variable, $\phi$ is the azimuthal angle and $z$ is the axial variable. The $\mathbf{E}$ is the axis of rotation for the system, and the $z$ axis is the axis of rotation for the liquid bridge. The liquid bridge spinning about the $z$ axis is subjected to a forced precession about the $\mathbf{E}$ axis. We assume that the rate of precession $\epsilon$ is small.

In a coordinate system rotating with a constant angular velocity about $\mathbf{E}$, the inviscid Euler equations require the instantaneous velocity field $\mathbf{v}$ to satisfy

$$
\frac{\partial \mathbf{v}}{\partial t}+\mathbf{v} \cdot \nabla \mathbf{v}+\mathbf{2} \epsilon \mathbf{E} \times \mathbf{v}=-\nabla \pi, \quad \nabla \cdot \mathbf{v}=\mathbf{0}
$$

where $\mathbf{E}=(\cos \phi,-\sin \phi, 0)$ and $\epsilon$ is the strength of external Coriolis force. These equations are supplemented with the kinematic and the dynamic boundary conditions. For $\epsilon=0$ we find that $\mathbf{V}(r)=(0, \Omega r, 0)$ is a solution. For $\epsilon=0$ the problem is axisymmetric i.e. invariant under all transformations belonging to the continuous group $S^{1}$ of rotations about the $z$ axis: $\phi \rightarrow \phi+\alpha$.

In the following we assume that the drop surfaces can be written in the form

$$
r=\zeta(\phi, z, t) .
$$

In this section only azimuthal wavenumbers will be denoted by $m$ ( $m=0$ corresponds to axisymmetric modes). The base state corresponding to a rigidly rotating 
cylindrical drop is

$$
\mathbf{U}=(0, \Omega r, 0), P=p_{\infty}+\frac{\gamma}{a}, \zeta=a
$$

where $p_{\infty}$ is the pressure outside the drop and $a$ is the drop radius. For the mean curvature we have

$$
\kappa=\nabla \cdot \mathbf{n}
$$

where $\mathbf{n}$ is unit outward normal of the drop surface and $\mathbf{n}$ is related to $\zeta$ by

$$
\mathbf{n}=\left(1,-\frac{1}{r} \frac{\partial \zeta}{\partial \phi},-\frac{\partial \zeta}{\partial z}\right) /\left(1+\left(\frac{1}{r} \frac{\partial \zeta}{\partial \phi}\right)^{2}+\left(\frac{\partial \zeta}{\partial z}\right)^{2}\right)^{1 / 2}
$$

The Euler equations, the kinematic and the dynamic boundary conditions are linearized in the disturbance field near the basic state (4.2). The linearized problem is supplemented with the linearized kinematic and dynamic boundary conditions

$$
u=\left.\frac{\partial \zeta}{\partial t}\right|_{r=a}, p=-\left.\gamma\left(\frac{\partial^{2} \zeta}{\partial z^{2}}+\frac{1}{a^{2}} \frac{\partial^{2} \zeta}{\partial \phi^{2}}+\frac{\zeta}{a^{2}}\right)\right|_{r=a} .
$$

The corresponding linear problem is solved numerically following Mahalov [1993]. In order to ensure zero flux boundary conditions in the vertical direction on the vertical boundaries, one only needs to restrict Fourier series to be even in $z$ for horizontal velocities and odd in $z$ for vertical velocity.

Breaking the rotational symmetry of the liquid bridge via precession will move double eigenvalues off the axis (see Guckenheimer and Mahalov [1992]; Knobloch, Mahalov and Marsden [1994]). Because of the Hamiltonian structure of the system the eigenvalues will move to both sides of the imaginary axis, thereby destabilizing the system. We can associate instabilities with the degeneracies caused by two physically distinguishable eigenmodes having the same eigenfrequency. The flow is unstable around the $m=0$ and $m=1$ double eigenvalue of the unperturbed rotating columnar liquid drop. Unstable vertical heights and growth rates resulting from splitting of double eigenvalues are given in Table 1.

\begin{tabular}{|l|l|l|l|}
\hline unstable vertical height $(\mathrm{h})$ & 7.156 & 5.796 & 5.262 \\
\hline growth rate & $0.549 \epsilon$ & $0.104 \epsilon$ & $0.054 \epsilon$ \\
\hline
\end{tabular}

Table 1. Unstable vertical heights and growth rates. 


\section{APPENDICES}

\section{A Linear Stability Analysis of Rotating Liquid Drops}

Consider a solution to the boundary value problem (3.1)-(3.2). This solution represents a drop rotating with constant angular velocity $\Omega$ about the $z$-axis. The free boundary $\Sigma$ of the drop has $f:[0, h] \rightarrow \mathbb{R}^{+}$as its profile curve. For a 3 -D linear stability analysis of this drop motion it turns out to be very convenient to choose a certain kind of coordinate system in a body frame (i.e. a frame attached to the rigidly rotating fluid mass). We assume there is an orthogonal coordinate system $\Psi:(R, \Phi, \Theta) \mapsto \mathbb{R}^{3}$ with the following properties:

- $\frac{\partial \Psi}{\partial R}(x)$ is an outer unit normal vector to $\Sigma$ at the point $x \in \Sigma$.

- $\frac{\partial \Psi}{\partial \Theta}(x)$ is a conormal vector to $\Sigma$ at $x \in c_{i}$, i.e. $\frac{\partial \Psi}{\partial \Theta}(x)$ is perpendicular to $T_{x} c_{i}$ and tangent to $\Sigma$ at $x, i=0,1$.

- $\Sigma$ is given in these coordinates by $R=0$.

- The unit vector $\mathbf{e}_{z}$ along the $z$-axis of the cartesian coordinate system previously introduced is perpendicular to

$$
\mathbf{e}_{\Phi}:=\frac{1}{\left\|\frac{\partial \Psi}{\partial \Phi}\right\|} \frac{\partial \Psi}{\partial \Phi} .
$$

- $\left\langle\frac{\partial \Psi}{\partial R}, \frac{\partial \Psi}{\partial R}\right\rangle=1$.

Thus, there are $d_{1}, d_{2} \in \mathbb{R}$ such that

$$
\mathbf{e}_{z}=d_{1} \mathbf{e}_{R}+d_{2} \mathbf{e}_{\theta}
$$

where

$$
\mathbf{e}_{R}:=\frac{\partial \Psi}{\partial R} /\left\|\frac{\partial \Psi}{\partial R}\right\|, \mathbf{e}_{\Theta}:=\frac{\partial \Psi}{\partial \Theta} /\left\|\frac{\partial \Psi}{\partial \Theta}\right\| .
$$

In the case of a cylindrical drop with radius $a$ we may choose $\Psi:(R, \Phi, \Theta) \mapsto$ $((R+a) \cos \Phi,(R+a) \sin \Phi, \Theta)$ in order to get a coordinate system satisfying the above conditions. In other cases the existence of coordinate systems with the listed properties is guaranteed by the theory of conformal mappings. Let $\left(g_{i j}\right)_{i, j=R, \Phi, \Theta}$ denote the metric tensor in the coordinates $R, \Phi, \Theta$ and

$$
D:=\sqrt{\operatorname{det}\left(g_{i j}\right)}
$$

Let

$$
\Theta-p^{i}(R, \Phi)=0
$$


be the parameterization of the plate $P_{i}, i=0,1$, in our coordinate system. We perform a linear stability analysis of our rigidly rotating drop. To linearize the equations of motion (2.1)-(2.6) about a stationary solution, given by a velocity field

$$
\mathbf{v}=u \mathbf{e}_{R}+v \mathbf{e}_{\Phi}+w \mathbf{e}_{\theta},
$$

a free surface $\Sigma$, defined by $R-\zeta(\Phi, \Theta)=0$ and a pressure field $p$, we set

$$
\begin{aligned}
\bar{u}=u+u^{\prime}+O\left(u^{\prime 2}\right), \bar{v} & =v+v^{\prime}+O\left(v^{\prime 2}\right), \bar{w}=w+w^{\prime}+O\left(w^{\prime 2}\right), \\
\bar{p} & =p+p^{\prime}+O\left(p^{\prime 2}\right), \bar{\zeta}=\zeta+\zeta^{\prime}+O\left(\zeta^{\prime 2}\right) .
\end{aligned}
$$

We substitute this into the equations of motion (2.1)-(2.6). Then we neglect higher order terms in the primed variables and make use of the fact that $u, v, w, p, \zeta$ is a solution to the equations of motion. In the case of our rigidly rotating drop we have $u=v=w=0$, because the drop is at rest in a rotating frame. Also, $\zeta=0$ in this case, as the free boundary of the rotating drop is given by $R=0$ by assumption about our coordinate system. The linearized equations of motion are in this case

$$
\begin{aligned}
\frac{\partial \mathbf{v}^{\prime}}{\partial t} & =-\nabla p^{\prime}-2 \Omega \mathbf{e}_{z} \times \mathbf{v}^{\prime}, & \nabla \cdot \mathbf{v}^{\prime}=0, & \\
\left\langle\mathbf{v}^{\prime}, \mathbf{e}_{z}\right\rangle & =0 & & \text { on } \Sigma_{0} \cup \Sigma_{1}, \\
\frac{\partial \zeta^{\prime}}{\partial t} & =u^{\prime} & & \text { on } \Sigma, \\
p^{\prime} & =\gamma\left(\frac{D_{R R}}{D}-\frac{D_{R}^{2}}{D^{2}}\right) \zeta^{\prime}-\gamma \triangle_{\Sigma} \zeta^{\prime} & & \text { on } \Sigma, \\
j_{i} \zeta^{\prime}+l_{i} \zeta_{\Theta}^{\prime} & =0 & & \text { on } c_{i}, i=0,1,
\end{aligned}
$$

where

$$
j_{i}=2 d_{1}\left(d_{2}\right)_{R}-\left(d_{1}\right)_{\Theta} p_{R}^{i}+\frac{d_{1}^{2}\left(d_{1} d_{2}\right)_{R}}{d_{2}}-\left(d_{1}\right)_{R}-\frac{d_{1}\left(d_{2}\right)_{R}}{d_{2}}, l_{i}=\frac{d_{2}}{\sqrt{g_{\Theta \Theta}}} .
$$

and $\triangle_{\Sigma}$ denotes the Laplacian on $\Sigma$. For a derivation of these equations compare the Appendix B.

In the following we drop the primes from the variables in equations (A.3)-(A.7). For the linear stability analysis we make the Ansatz

$$
\begin{gathered}
\mathbf{v}(R, \Phi, \Theta, t)=e^{i(n \Phi-\omega t)} \hat{\mathbf{v}}(R, \Theta) \\
p(R, \Phi, \Theta, t)=e^{i(n \Phi-\omega t)} \hat{p}(R, \Theta), \zeta(\Phi, \Theta, t)=e^{i(n \Phi-\omega t)} \hat{\zeta}(\Theta) .
\end{gathered}
$$

If there are solutions to equations (A.3)-(A.7) of this form with $\operatorname{Im}(\omega)>0$, then the rotational motion is unstable. With the Ansatz (A.8)-(A.9) the system (A.3)-(A.7) can be reduced to an equivalent system for the variation of the pressure field $p$ and the variation $\zeta: \Sigma \rightarrow \mathbb{R}$ of the free boundary $\Sigma$ only. We write down this system 
only for the case $\Omega=0$. In this case the equations have a particularly simple form (the general case is treated in Section 4.1):

$$
\begin{aligned}
\triangle p & =0 & & \text { in } D_{\Sigma}, \\
\langle\nabla p, \mathbf{n}\rangle & =0 & & \text { on } \Sigma_{0} \cup \Sigma_{1}, \\
p_{R} & =\omega^{2} \zeta & & \text { on } \Sigma, \\
p & =\gamma\left(\frac{D_{R R}}{D}-\frac{D_{R}^{2}}{D^{2}}\right) \zeta-\gamma \triangle_{\Sigma} \zeta & & \text { on } \Sigma, \\
j_{i} \zeta+l_{i} \zeta_{\Theta} & =0 & & \text { on } c_{i}, i=0,1,
\end{aligned}
$$

where $D_{\Sigma}$ denotes the region enclosed by $\Sigma$ and the two plates. (Observe that the equations (A.13), (A.14) are just the boundary conditions (A.6) and (A.7).) Now we present a technique to solve this boundary value problem. Let

$$
L(\zeta):=\gamma\left(\frac{D_{R R}}{D}-\frac{D_{R}^{2}}{D^{2}}\right) \zeta-\gamma \triangle_{\Sigma} \zeta
$$

The operator $L$ is a symmetric operator on functions $f: \Sigma \rightarrow \mathbb{R}$ with appropriate differentiability properties, which satisfy the boundary condition (A.14) and the condition $\int_{\Sigma} f d S=0$. This follows by applying Green's identity on the manifold with boundary $\Sigma$ and remembering that $\mathbf{e}_{\Theta}$ is a conormal field to $\Sigma$ on the boundary of $\Sigma$. We assume that $L$ has a discrete set of eigenvalues $\lambda_{i}, i=1,2, \ldots$ with corresponding eigenfunctions $\zeta_{i}, i=1,2, \ldots$ For $i=1,2, \ldots$ let $q_{i}: D_{\Sigma} \rightarrow \mathbb{R}$ be the solution of the Neumann problem

$$
\begin{aligned}
\triangle q_{i} & =0, & & \\
\left\langle\nabla q_{i}, \mathbf{e}_{z}\right\rangle & =0 & & \text { on } \Sigma_{0} \cup \Sigma_{1}, \\
\left(q_{i}\right)_{R} & =\zeta_{i} & & \text { on } \Sigma .
\end{aligned}
$$

The assumption $\int_{\Sigma} \zeta_{i} d S=0$ guarantees the solvability of this problem. Note that this assumption corresponds to the fact that we consider an inviscid liquid drop. Let

$$
q_{i}(x)=\sum_{j=1}^{\infty} e_{i j} \zeta_{j}(x)
$$

for $x \in \Sigma$. We make the following Ansatz for the solution $p: D_{\Sigma} \rightarrow \mathbb{R}, \zeta: \Sigma \rightarrow \mathbb{R}$ for the system (A.10)-(A.14):

$$
\begin{aligned}
p & =\sum_{i=0}^{\infty} c_{i} q_{i}, \\
\zeta(x) & =-\frac{1}{\omega^{2}} p_{R}(x) \quad \text { for } x \in \Sigma .
\end{aligned}
$$

Then (A.10)-(A.12) and (A.14) are satisfied by construction. The left side of equation (A.13) reads

$$
p(x)=\sum_{i=1}^{\infty} c_{i} q_{i}(x)=\sum_{i=1}^{\infty}\left(\sum_{j=1}^{\infty} c_{j} e_{j i}\right) \zeta_{i} .
$$


The right hand side reads

$$
-\frac{\gamma}{\omega^{2}}\left(\frac{D_{R R}}{D}-\frac{D_{R}^{2}}{D^{2}}\right) p_{R}+\frac{\gamma}{\omega^{2}} \triangle_{\Sigma} p_{R}=-\frac{1}{\omega^{2}} L\left(\sum_{i=1}^{\infty} c_{i} \zeta_{i}\right)=-\sum_{i=1}^{\infty} \frac{c_{i} \lambda_{i}}{\omega^{2}} \zeta_{i} .
$$

Equating (A.20) and (A.21) yields

$$
\sum_{j=1}^{\infty} e_{j i} c_{j}=-\frac{\lambda_{i}}{\omega^{2}} c_{i}
$$

for $i=1,2, \ldots$ The real numbers $c_{i}, i=1,2, \ldots$ and $\omega$ have to be determined from Equation (A.22).

These numbers can be obtained easily in the case that solutions $q_{i}$ to the Neumann problem (A.16)-(A.18) can be found by separation of variables in the form

$$
q_{i}(R, \Phi, \Theta)=r_{i}(R) s_{i}(\Phi, \Theta) .
$$

By definition of $q_{i}$ and because the surface $\Sigma$ is given by $R=0$, we have

$$
\left(r_{i}\right)_{R}(0) s_{i}(\Phi, \Theta)=\left(q_{i}\right)_{R}(0, \Phi, \Theta)=\zeta_{i}(\Phi, \Theta) .
$$

Setting $a_{i}:=r_{i}(0)$ and $b_{i}:=\left(r_{i}\right)_{R}(0)$, we then obtain

$$
q_{i}(0, \Phi, \Theta)=\frac{a_{i}}{b_{i}} \zeta_{i}(\Phi, \Theta) \text {. }
$$

Thus,

$$
e_{i j}=\delta_{i j} \frac{a_{i}}{b_{i}}
$$

and equation (A.22) becomes

$$
\frac{a_{i}}{b_{i}} c_{i}=\frac{\lambda_{i}}{\omega^{2}} c_{i}
$$

for $i=1,2, \ldots$ Choose $c_{1}=1, \omega^{2}=\frac{b_{1} \lambda_{1}}{a_{1}}$ and $c_{i}=0$ for $i \neq 1$. Solutions to the equations (A.10)-(A.14) in the form (A.23) can be obtained for instance in the case that the rotating drop whose stability is analyzed has a cylindrical shape. Such solutions to the equations of motion (2.1)-(2.6) exist, if $\sigma_{i}=0$ for $i=0,1$.

In section 5 we will use separation of variables to solve the linear stability problem for a rotating liquid column analytically. In the case $\Omega \neq 0$ one can still reduce the linearized equations of motion (A.3)-(A.7) to a system for the variation of the pressure and the free boundary. In this case one has to deal with a mixed boundary value problem for a second order operator with variable coefficients. We observe that the linearized dynamic boundary conditions (A.6) and the linearized contact angle conditions (A.7) stay the same if $\Omega \neq 0$. So the solution technique presented above can be applied after some modifications. We will discuss the case $\Omega \neq 0$ in the following section. 


\section{A.1 The Treatment of the Case $\Omega \neq 0$}

Now we consider the case $\Omega \neq 0$. We start from the linearized equations of motion (A.3)-(A.7). For $\mathbf{v}=(u, v, w), p$ we make the Ansatz (A.8)-(A.9). In our coordinates the equations (A.3) read as follows:

$$
\begin{aligned}
-i \omega u & =-\frac{\partial p}{\partial R}-\epsilon d_{2} v \\
-i \omega v & =-\frac{i n}{\sqrt{g_{\Phi \Phi}}} p+\epsilon d_{2} u-\epsilon d_{1} w \\
-i \omega w & =-\frac{1}{\sqrt{g_{\Theta \Theta}}} \frac{\partial p}{\partial \Theta}+\epsilon d_{1} v \\
0 & =\frac{\partial}{\partial R}(D u)+\frac{\partial}{\partial \Phi}\left(\frac{D}{\sqrt{g_{\Phi \Phi}}} v\right)+\frac{\partial}{\partial \Theta}\left(\frac{D}{\sqrt{g_{\Theta \Theta}}} w\right) .
\end{aligned}
$$

Here $D, d_{1}, d_{2}$ are as in equations (A.1) and (A.2), and $\epsilon= \pm 2|\Omega|$. We choose the plus sign if $\Phi$ is measured clockwise and the minus sign if $\Phi$ is measured counterclockwise. After a long but straightforward calculation we get from equations (A.24)-(A.25), making use of the fact that $d_{1}^{2}+d_{2}^{2}=1$,

$$
\begin{aligned}
u= & \frac{i}{\omega} \cdot \frac{\omega^{2}-\epsilon^{2} d_{1}^{2}}{\epsilon^{2}-\omega^{2}} \frac{\partial p}{\partial R}+\frac{i n \epsilon d_{2}}{\sqrt{g_{\Phi \Phi}}\left(\epsilon^{2}-\omega^{2}\right)} p-\frac{i \epsilon^{2} d_{1} d_{2}}{\omega \sqrt{g_{\Theta \Theta}}\left(\epsilon^{2}-\omega^{2}\right)} \frac{\partial p}{\partial \Theta}, \\
v= & -\frac{\omega n}{\sqrt{g_{\Phi \Phi}}\left(\epsilon^{2}-\omega^{2}\right)} p+\frac{\epsilon d_{1}}{\sqrt{g_{\Theta \Theta}}\left(\epsilon^{2}-\omega^{2}\right)} \frac{\partial p}{\partial \Theta}-\frac{\epsilon d_{2}}{\epsilon^{2}-\omega^{2}} \frac{\partial p}{\partial R} \\
w= & -\frac{i \epsilon^{2} d_{1} d_{2}}{\omega\left(\epsilon^{2}-\omega^{2}\right)} \frac{\partial p}{\partial R}-\frac{i \epsilon d_{1} n}{\sqrt{g_{\Phi \Phi}}\left(\epsilon^{2}-\omega^{2}\right)} p+\frac{i\left(\omega^{2}-\epsilon^{2} d_{2}^{2}\right)}{\omega \sqrt{g_{\Theta \Theta}}\left(\epsilon^{2}-\omega^{2}\right)} \frac{\partial p}{\partial \Theta}, \\
0= & \omega^{2} \triangle p-\frac{\epsilon^{2}}{D} \frac{\partial}{\partial R}\left(D d_{1}^{2} \frac{\partial p}{\partial R}\right)-\frac{\epsilon^{2}}{D} \frac{\partial}{\partial \Theta}\left(\frac{\sqrt{g_{\Phi \Phi}}}{\sqrt{g_{\Theta \Theta}}} d_{2}^{2} \frac{\partial p}{\partial \Theta}\right) \\
& -\frac{\epsilon^{2}}{D}\left[\frac{\partial}{\partial R}\left(d_{1} d_{2} \sqrt{g_{\Phi \Phi}} \frac{\partial p}{\partial \Theta}\right)+\frac{\partial}{\partial \Theta}\left(d_{1} d_{2} \sqrt{g_{\Phi \Phi}} \frac{\partial p}{\partial R}\right)\right] \\
& +\frac{n \omega \epsilon}{D} p\left(\frac{\partial}{\partial R}\left(d_{2} \sqrt{g_{\Theta \Theta}}\right)\right)-\frac{n \omega \epsilon}{D}\left[d_{1} \frac{\partial p}{\partial \Theta}+\frac{\partial}{\partial \Theta}\left(d_{1} p\right)\right]
\end{aligned}
$$

Now observe that in the derivation of these equations we only used properties of the coordinate system which are also satisfied by cylindrical coordinates. Thus, we may choose at this point - where we do not consider boundary conditions - cylindrical coordinates $(R, \Phi, \Theta)=(R, \Phi, z)$. Then we have $D=R, g_{\Phi \Phi}=R^{2}, g_{R R}=1$, and $g_{\Theta \Theta}=1$. Furthermore, $d_{1}=0$ and $d_{2}=1$ hold and from equation (A.29) we get

$$
0=\omega^{2} \triangle p-\epsilon^{2} \frac{\partial^{2} p}{\partial z^{2}}
$$

¿From the equations (A.29) and (A.30) we deduce that the term added to $\omega^{2} \Delta p$ on the right hand side of (A.29) is nothing but $-\epsilon^{2} \frac{\partial^{2} p}{\partial z^{2}}$. 
Now we look at the boundary conditions to the equations (A.24)-(A.25) in the special coordinate system described in the last section. The boundary conditions are given in equations (A.4)-(A.7). ¿From equations (A.26) and (A.5) we get

$$
\zeta=\frac{1}{\omega^{2}-\epsilon^{2}} \frac{\partial p}{\partial R}-\frac{\epsilon^{2} d_{1}}{\omega^{2}\left(\omega^{2}-\epsilon^{2}\right)} \frac{\partial p}{\partial z}+\frac{\epsilon n d_{2}}{\sqrt{g_{\Phi \Phi}} \omega\left(\omega^{2}-\epsilon^{2}\right)} p
$$

on the free boundary $\Sigma$. Here we used the fact that

$$
\frac{\partial p}{\partial z}=d_{1} \frac{\partial p}{\partial R}+\frac{d_{2}}{\sqrt{g_{\Theta \Theta}}} \frac{\partial p}{\partial \Theta} .
$$

The kinematic boundary condition (A.4) at the plates can be written as

$$
d_{1} u+d_{2} w=0 \quad \text { on } \Sigma_{0} \cup \Sigma_{1} .
$$

Because of equations (A.26), (A.28), and (A.31), equation (A.32) can be written as

$$
\frac{\partial p}{\partial z}=0 \quad \text { on } \Sigma_{0} \cup \Sigma_{1} .
$$

Now we extend the solution technique introduced in the last section to the case $\Omega \neq 0$. Equations (A.16)-(A.18) have to be substituted in the general case by the equations

$$
\begin{aligned}
0 & =\omega^{2} \triangle q_{i}-\epsilon^{2} \frac{\partial^{2} q_{i}}{\partial z^{2}} \\
\zeta_{i} & =\frac{1}{\omega^{2}-\epsilon^{2}} \frac{\partial q_{i}}{\partial R}-\frac{\epsilon^{2} d_{1}}{\omega^{2}\left(\omega^{2}-\epsilon^{2}\right)} \frac{\partial q_{i}}{\partial z}+\frac{\epsilon n d_{2}}{\sqrt{g_{\Phi \Phi}} \omega\left(\omega^{2}-\epsilon^{2}\right)} q_{i} \\
\frac{\partial q_{i}}{\partial z} & =0 \quad \text { on } \Sigma_{0} \cup \Sigma_{1} .
\end{aligned}
$$

Here $\zeta_{i}, i=1,2, \ldots$ denotes an orthonormal basis of eigenfunctions of the operator $L$ introduced in the last section. As in (A.19) we expand

$$
q_{i}(x)=\sum_{j=1}^{\infty} e_{i j} \zeta_{j}(x),
$$

for $x \in \Sigma$. Thus,

$$
e_{i j}=\int_{\Sigma} q_{i} \zeta_{j} d S
$$

In Appendix $\mathrm{C}$ we show the self-adjointness property

$$
e_{i j}=e_{j i} .
$$

for $i, j \in \mathbb{N}$. We now make the Ansatz $p=\sum_{i=0}^{\infty} c_{i} q_{i}$ and

$$
\zeta=\frac{1}{\omega^{2}-\epsilon^{2}} \frac{\partial p}{\partial R}-\frac{\epsilon^{2} d_{1}}{\omega^{2}\left(\omega^{2}-\epsilon^{2}\right)} \frac{\partial p}{\partial z}+\frac{\epsilon n d_{2}}{\sqrt{g_{\Phi \Phi}} \omega\left(\omega^{2}-\epsilon^{2}\right)} p \quad \text { on } \Sigma,
$$

and proceed as in the previous section. 


\section{B Derivation of the Linearized Boundary Conditions in the Special Coordinate System}

In this Appendix we present a detailed derivation of the boundary conditions in the coordinate system introduced in Section 4.

To derive the linearized dynamic boundary condition (A.6) we make use of the fact that the mean curvature $\kappa: \bar{\Sigma} \rightarrow \mathbb{R}$ of a surface $\bar{\Sigma} \subseteq \mathbb{R}^{3}$ can be written as

$$
\kappa=\nabla \cdot \overline{\mathbf{n}},
$$

where $\overline{\mathbf{n}}$ denotes an outer unit normal vector field to $\bar{\Sigma}$. We regard $\bar{\Sigma}$ as a disturbance of $\Sigma$. Barred variables refer to geometric data of $\bar{\Sigma}$ whereas unbarred variables refer to those of $\Sigma$.

If $\bar{\Sigma}$ is defined by the equation $R-\bar{\xi}=0$, then

$$
\kappa=\nabla \cdot\left(\frac{\nabla(R-\bar{\zeta})}{\|\nabla(R-\bar{\zeta})\|}\right) .
$$

Now, set $\bar{\zeta}=\zeta^{\prime}+O\left(\zeta^{\prime 2}\right)$ (recall that the surface $\Sigma$ about which we linearize is given by $R=0$ ), expand the right side of (B.1) in orders of $\zeta^{\prime}$ and neglect terms of order $O\left(\zeta^{\prime 2}\right)$. To express the linearized mean curvature operator with the help of the Laplacian $\triangle_{\Sigma}$ on the surface $\Sigma$ use the fact that $g_{R R}=1$ by our assumption about the coordinate system.

To derive the linearized contact angle condition (A.7) observe that a tangential vector field to the contact curve $\bar{c}_{i}$ of $\bar{\Sigma}$ with the plate $P_{i}, i=0,1$ is given by

$$
\overline{\mathbf{t}}_{i}=\frac{d_{2} \zeta_{\Phi}^{\prime}}{\sqrt{g_{\Phi \Phi}}} \mathbf{e}_{R}+\left(d_{2}+\frac{d_{1} \zeta_{\Theta}^{\prime}}{\sqrt{g_{\Theta \Theta}}}\right) \mathbf{e}_{\Phi}-\left(\frac{d_{1} \zeta_{\phi}^{\prime}}{\sqrt{g_{\Phi \Phi}}}+d_{2}\right) \mathbf{e}_{\theta}+O\left(\zeta^{\prime 2}\right)
$$

We get this tangential vector field simply by taking the cross product of an outer normal vector to $\bar{\Sigma}$ and the vector $\mathbf{e}_{z}$ at every point $x \in \bar{c}_{i}$. A normal vector field to $\bar{c}_{i}$ (regarded as a submanifold of the plate $P_{i}$ ) is given by $\overline{\mathbf{n}}_{i}=\overline{\mathbf{t}}_{i} \times \mathbf{e}_{z}$, i.e.

$$
\begin{aligned}
\overline{\mathbf{n}}_{i}= & \left(d_{2}^{2}+\frac{d_{1} d_{2} \zeta_{\Theta}^{\prime}}{\sqrt{g_{\Theta \Theta}}}\right) \mathbf{e}_{R}-\left(\frac{d_{1}^{2} \zeta_{\Phi}^{\prime}}{\sqrt{g_{\Phi \Phi}}}+\frac{d_{2}^{2} \zeta_{\Phi}^{\prime}}{\sqrt{g_{\Phi \Phi}}}\right) \mathbf{e}_{\Phi} \\
& -\left(d_{1} d_{2}+\frac{d_{1}^{2} \zeta_{\Theta}^{\prime}}{\sqrt{g_{\Theta \Theta}}}\right) \mathbf{e}_{\theta}+O\left(\zeta^{\prime 2}\right) .
\end{aligned}
$$

A conormal vector field to $\bar{\Sigma}$ on $\bar{c}_{i}$ is given by $\overline{\mathbf{k}}_{i}=\overline{\mathbf{n}} \times \overline{\mathbf{t}}_{i}$, i.e.

$$
\overline{\mathbf{k}}_{i}=\frac{d_{2} \zeta_{\Theta}^{\prime}}{\sqrt{g_{\Theta \Theta}}} \mathbf{e}_{R}+\frac{d_{1} \zeta_{\Phi}^{\prime}}{\sqrt{g_{\Phi \Phi}}} \mathbf{e}_{\Phi}+\left(\frac{d_{1} \zeta_{\Theta}^{\prime}}{\sqrt{g_{\Theta \Theta}}}+d_{2}\right) \mathbf{e}_{\theta}+O\left(\zeta^{\prime 2}\right)
$$

Thus, we have for the cosine of the contact angle $\bar{\alpha}_{i}$ in which the surface $\bar{\Sigma}$ meets the plate $P_{i}$

$$
\begin{aligned}
\frac{\left\langle\overline{\mathbf{n}}_{i}, \overline{\mathbf{k}}_{i}\right\rangle}{\left\|\overline{\mathbf{n}}_{i}\right\|\left\|\overline{\mathbf{k}}_{i}\right\|}= & -d_{1}+\left(2 d_{1}\left(d_{2}\right)_{R}+\frac{d_{1}^{2}\left(d_{1} d_{2}\right)_{R}}{d_{2}}-\left(d_{1}\right)_{R}-\frac{d_{1}\left(d_{2}\right)_{R}}{d_{2}}\right) \zeta^{\prime} \\
& +\frac{d_{2}}{\sqrt{g_{\Theta \Theta}}} \zeta_{\Theta}^{\prime}+O\left(\zeta^{\prime 2}\right),
\end{aligned}
$$


where the terms $d_{1}, d_{2}, g_{\Theta \Theta}$ have to be evaluated on the curve $\bar{c}_{i}, i=0,1$. By assumption, the plate $P_{i}$ is given by the equation $\Theta-p^{i}(R, \Phi)=0$ in our coordinate system. Now

$$
p^{i}(\bar{\zeta}, \Phi)=p^{i}(0, \Phi)+p_{R}^{i}(0, \Phi) \zeta^{\prime}+O\left(\zeta^{\prime 2}\right) .
$$

Use (B.3) in (B.2) to obtain

$$
\begin{aligned}
\frac{\left\langle\overline{\mathbf{n}}_{i}, \overline{\mathbf{k}}_{i}\right\rangle}{\left\|\overline{\mathbf{n}}_{i}\right\|\left\|\overline{\mathbf{k}}_{i}\right\|}= & -d_{1}+\left(2 d_{1}\left(d_{2}\right)_{R}+\frac{d_{1}^{2}\left(d_{1} d_{2}\right)_{R}}{d_{2}}-\left(d_{1}\right)_{R}-\left(d_{1}\right)_{\Theta} p_{R}^{i}-\frac{d_{1}\left(d_{2}\right)_{R}}{d_{2}}\right) \zeta^{\prime} \\
& +\frac{d_{2}}{\sqrt{g_{\Theta \Theta}}} \zeta_{\Theta}+O\left(\zeta^{\prime 2}\right),
\end{aligned}
$$

where the quantities $p_{R}^{i}, d_{1}, d_{2}$ on the right side of equation (B.4) have to be evaluated at the unperturbed contact curve $c_{i}$.

In the same way one derives the linearized kinematic boundary condition (A.5) from the nonlinear one (2.4), which reads in our coordinate system as

$$
\langle\overline{\mathbf{v}}, \overline{\mathbf{n}}\rangle=\frac{\partial \bar{\zeta}}{\partial t}
$$

\section{Self-Adjointness Properties Arising in the Stability Problem}

In this part of the Appendix we prove the self-adjointness property

$$
e_{i j}=e_{j i}
$$

for $i, j \in \mathbb{N}$, stated in Eq. (A.36) of the main text. By Eq. (A.34) we have

$$
\int_{\Sigma} q_{i} \zeta_{j} d S=A+B+C
$$

where

$$
A:=\int_{\Sigma} \frac{q_{i}}{\omega^{2}-\epsilon^{2}} \frac{\partial q_{j}}{\partial R} d S, B:=\int_{\Sigma} \frac{-\epsilon^{2} q_{i} d_{1}}{\omega^{2}\left(\omega^{2}-\epsilon^{2}\right)} \frac{\partial q_{j}}{\partial z} d S, C:=\int_{\Sigma} \frac{\epsilon n d_{2} q_{i}}{\sqrt{g_{\Phi \Phi}} \omega\left(\omega^{2}-\epsilon^{2}\right)} q_{j} d S .
$$

The term $C$ is symmetric in $i$ and $j$. We only have to check that $A+B$ is symmetric in $i$ an $j$. By equation (A.35) and the divergence theorem, we have

$$
A=\int_{D_{\Sigma}} \frac{\operatorname{div}\left(q_{i} \nabla q_{j}\right)}{\omega^{2}-\epsilon^{2}} d V=A_{1}+A_{2}
$$

where

$$
A_{1}:=\int_{D_{\Sigma}} \frac{\left\langle\nabla q_{i}, \nabla q_{j}\right\rangle}{\omega^{2}-\epsilon^{2}} d V, A_{2}:=\int_{D_{\Sigma}} \frac{q_{i} \triangle q_{j}}{\omega^{2}-\epsilon^{2}} d V
$$

The term $A_{1}$ is symmetric in $i$ and $j$. It remains to show that $A_{2}+B$ is symmetric in $i$ and $j$. 
By equation (A.33) we have

$$
A_{2}=\int_{D_{\Sigma}} \frac{\epsilon^{2}}{\omega^{2}\left(\omega^{2}-\epsilon^{2}\right)} q_{i} \frac{\partial^{2} q_{j}}{\partial z^{2}} d V .
$$

Because $d_{1}=\left\langle e_{z}, e_{R}\right\rangle$, we obtain (using equation (A.35) and the divergence theorem)

$$
\begin{aligned}
B & =-\int_{\Sigma} \frac{\epsilon^{2} q_{i}\left\langle e_{z}, e_{R}\right\rangle}{\omega^{2}\left(\omega^{2}-\epsilon^{2}\right)} \frac{\partial q_{j}}{\partial z} d S \\
& =-\int_{D_{\Sigma}} \frac{\epsilon^{2}}{\omega^{2}\left(\omega^{2}-\epsilon^{2}\right)} \operatorname{div}\left(\frac{\partial q_{j}}{\partial z} q_{i} e_{z}\right) d V \\
& =-\int_{D_{\Sigma}} \frac{\epsilon^{2}}{\omega^{2}\left(\omega^{2}-\epsilon^{2}\right)}\left\langle\nabla q_{i}, e_{z}\right\rangle \frac{\partial q_{j}}{\partial z} d V-\int_{D_{\Sigma}} \frac{\epsilon^{2}}{\omega^{2}\left(\omega^{2}-\epsilon^{2}\right)} q_{i} \operatorname{div}\left(\frac{\partial q_{j}}{\partial z} e_{z}\right) d V \\
& =-\int_{D_{\Sigma}} \frac{\epsilon^{2}}{\omega^{2}\left(\omega^{2}-\epsilon^{2}\right)} \frac{\partial q_{i}}{\partial z} \frac{\partial q_{j}}{\partial z} d V-\int_{D_{\Sigma}} \frac{\epsilon^{2}}{\omega^{2}\left(\omega^{2}-\epsilon^{2}\right)} q_{i} \frac{\partial^{2} q_{j}}{\partial z^{2}} d V
\end{aligned}
$$

Thus,

$$
A_{2}+B=-\int_{D_{\Sigma}} \frac{\epsilon^{2}}{\omega^{2}\left(\omega^{2}-\epsilon^{2}\right)} \frac{\partial q_{i}}{\partial z} \frac{\partial q_{j}}{\partial z} d V
$$

which is symmetric in $i$ and $j$. This proves the self-adjointness property.

Acknowledgments. We are grateful to Edgar Knobloch and Jürgen Scheurle for helpful comments and their interest. The research of HPK was partially supported by the DFG under the contract Sch $233 / 3-2$ as well as a former Feodor LynenFellowship of the Alexander von Humboldt-Foundation during a stay at the Fields Institute and the Department of Mathematics, University of California, Berkeley. That of AM was partially supported by AFOSR grant F49620-93-1-0172 and the ASU Center for Environmental Fluid Dynamics and that of JEM was partially supported by the Department of Energy and the California Institute of Technology.

\section{References}

[1] U. Abresch [1987], Constant mean curvature tori in terms of elliptic functions, Journ. f. Reine u. Angew. Math., 374, p. 169-192.

[2] A.I. Bobenko [1991], All constant mean curvature tori in $R^{3}, S^{3}, H^{3}$ in terms of theta-functions, Math. Ann., 290, p. 209-243.

[3] M. do Carmo [1976], Differential geometry of curves and surfaces, Englewood Cliffs, N.J., Prentice-Hall.

[4] W.C. Carter [1988], The forces and the behavior of fluids constrained by solids, Acta Metal., 36, p. 2283-2292.

[5] S. Chandrasekhar [1981], Hydrodynamic and Hydromagnetic Stability, Dover. 
[6] A. Davey and H. Salwen [1994], On the stability of flow in an elliptic pipe which is nearly circular, J. Fluid Mech., 281, p. 357-369.

[7] D.E. Delaunay [1841], Sur la surface de revolution dont la courbure est constante, J. Math. Pures Appl., 6, p. 309-315.

[8] M. Dellnitz, J.E. Marsden and I. Melbourne [1992], Generic bifurcation of Hamiltonian vector fields with symmetry, Nonlinearity, 5, p. 979.

[9] E. Doedel and X. Wang [1994], AUTO 94. Software for continuation and bifurcation problems in ordinary differential equations, California Institute of Technology.

[10] Drazin, P.G. and Reid, W.H. (1981), Hydrodynamic Stability, Cambridge University Press.

[11] R. Finn [1986], Equilibrium Capillary Surfaces, Springer, New York.

[12] R. Finn and T.I. Vogel [1992], On the volume infimum for liquid bridges, $Z$. Anal. Anw., 11, p. 3-23.

[13] J. Guckenheimer and A. Mahalov [1992], Instability induced by symmetry reduction, Phys. Rev. Lett., 68, No. 15, p. 2257.

[14] L.M. Hocking [1992], Rival contact-angle models and the spreading of drops, J. Fluid. Mech., 239, p. 671-681.

[15] J. Jost [1995] Riemannian Geometry and Geometric Analysis, Springer, Berlin.

[16] E. Knobloch, A. Mahalov and J.E. Marsden [1994], Normal forms for threedimensional parametric instabilities in ideal hydrodynamics, Physica D, 73, p. 49-81.

[17] D. Kröner [1987], The flow of a fluid with a free boundary and dynamic contact angle, Z. Angew. Math. Mech., 67, p. 304-306.

[18] H.-P. Kruse [1993], The Hamiltonian structure of the equations of motion of a liquid drop trapped between two plates, submitted for publication.

[19] H.-P. Kruse, J.E. Marsden and J. Scheurle [1993], On uniformly rotating fluid drops trapped between two parallel plates, in Exploiting Symmetry in Applied and Numerical Analysis, 1992 AMS-SIAM Summer Seminar in Applied Mathematics, July 26-August 1, 1992, Colorado State University, eds. E.L. Allgower, K. Georg, R. Miranda, Providence, Rhode Island, American Mathematical Society.

[20] L.D. Landau and E.M. Lifshitz [1958] Statistical Physics, Pergamon Press, London.

[21] D. Lewis, J.E. Marsden, R. Montgomery and T. Ratiu [1986], The Hamiltonian structure for dynamic free boundary value problems, Physica D, 18, p. 391-404. 
[22] D. Lewis, J.E. Marsden and T.S. Ratiu [1987], Stability and bifurcation of a rotating liquid drop, J. Math. Phys., 28, p. 2508.

[23] D. Lewis [1989], Nonlinear stability of a rotating planar liquid drop, Arch. Rat. Mech. and Anal., 106, p. 287.

[24] A. Mahalov [1993], The instability of rotating fluid columns subjected to a weak external Coriolis field, Phys. Fluids A, 5, p. 891-900.

[25] J.E. Marsden [1992], Lectures on Mechanics, London Math. Soc. Lect. Note Ser., 174, Cambridge University Press, Cambridge.

[26] A.D. Myshkis, V.G. Babskii, N.D. Kopachevskii, L.A. Slobozhanin, and A.D. Tyuptsov [1987], Low-Gravity Fluid Mechanics, Springer, Berlin.

[27] V.V. Pukhnachev and V.A. Solonnikov [1983], On the problem of dynamic contact angle, J. Appl. Math. Mech. (PMM), 46(6), 771-779.

[28] V.A. Solonnikov [1995], On some free boundary problems for the Navier-Stokes equations with moving contact points and lines, Math. Ann., 302, 743-772.

[29] M. Spivak [1979], A Comprehensive Introduction to Differential Geometry, 4, 2nd edition, Publish or Perish, Inc., Berkeley.

[30] J. Spruck [1988], The elliptic Sinh-Gordon equation and the construction of toroidal soap bubbles, in Calculus of Variations and Partial Differential Equations, eds.S. Hildebrandt, D. Kinderlehrer M. Miranda, Lect. Notes Math. 1340, Springer, New York.

[31] T.I. Vogel [1987], Stability of a liquid drop trapped between two parallel planes, SIAM J. Appl. Math, 47, p. 516-525.

[32] T.I. Vogel [1989], Stability of a liquid drop trapped between two planes II: general contact angles, SIAM J. Appl. Math., 49, p. 1009-1028.

[33] L. Zhou [1993], On the volume infimum for liquid bridges, Z. Anal. Anw., 12, p. 629-642. 